\section{Stability Ratios for Doublet Formation and for Deposition of Colloidal Particles with Arbitrary Interaction Potentials: An Analytical Approximation}

Thomas Gisler* and Michal Borkovec

Institute of Terrestrial Ecology, Federal Institute of Technology (ETH), Grabenstrasse 3, CH-8952 Schlieren, Switzerland

Received February 3, 1993. In Final Form: May 12, 1993

Unlike in fast (diffusion-limited) aggregation, the rate of doublet formation in slow (reaction-limited) coagulation is intimately linked to the details of the interaction potential between the colliding monomers. The classical Derjaguin-Landau-Verwey-Overbeek (DLVO) theory states that this total interaction potential ${ }^{1}$ can be expressed as a sum of two contributions, namely

$$
V(h)=U_{\mathrm{W}}(h)+U(h)
$$

where $h$ is the distance between the surfaces of the particles. The first contribution $U_{\mathrm{W}}$ is due to van der Waals attraction which can be approximated for spherical particles as $U_{\mathrm{W}}(h)=-A a / 12 h$ for $h \ll a$, where $a$ is the particle radius and $A$ the Hamaker constant. The second contribution is the electrostatic potential $U(h)$ which depends on parameters such as the charge $Z$ and the size of the particles, its surface potential $\Phi_{\mathrm{g}}$, and the inverse Debye screening length $\kappa$. The overall potential $V(h)$ itself has a maximum at $h=h_{\mathrm{g}}$ for ionic strengths below the critical coagulation concentration.

The doublet formation rate constant $k$ is defined by Smoluchowski's equation ${ }^{2} \mathrm{~d} c_{2} / \mathrm{d} t=k c_{1}{ }^{2}$, where $c_{1}$ and $c_{2}$ are the number densities of monomers and dimers, respectively. In the limit of fast aggregation where $V(h)$ $=0$, the rate constant is given by $k^{\text {fast }}=8 \pi a D$, where $D$ is the single particle diffusion coefficient. The actual coagulation rate constant $k^{\text {slow }}$ is commonly expressed in terms of the stability ratio $W=k^{\text {fast }} / k^{\text {slow. }}$. When the dynamics of the motion of the spherical particles is described by pure diffusion, Fuchs has shown that ${ }^{1,3}$

$$
W=2 a \int_{0}^{\infty} \mathrm{e}^{\beta V(h)}(h+2 a)^{-2} \mathrm{~d} h
$$

where $1 / \beta=k_{\mathrm{B}} T$ is the thermal energy unit. In the slow aggregation regime the structure of the DLVO potentials makes the integrand in eq 2 a sharply peaked function, and therefore only values in the neighborhood of the maximum of the potential $V(h)$ at $h=h_{\mathrm{s}}$ contribute to the integral. In this case a steepest descent approximation allows eq 2 to be written as a Gaussian integral giving the result 4

$$
W \simeq \pi^{1 / 2} \mathrm{e}^{\beta V\left(h_{\mathrm{v}}\right)}\left(2 a^{2} \beta\left|\partial^{2} V / \partial h^{2}\right|_{h=h_{\mathrm{e}}}\right)^{-1 / 2}
$$

We have used the fact that $a_{\kappa} \gg 1$ for all realistic systems. In order to proceed, one has to solve for the barrier location $h_{\mathrm{s}}(k)$ given by

$$
\left.\frac{\partial V(h)}{\partial h}\right|_{h}=0
$$

(1) Russel, W. B.; Saville, D. A.; Schowalter, W. R. Colloidal Dispersions; Cambridge University Press: Cambridge, 1989.

(2) von Smoluchowski, M. Z. Phys. Chem. 1917, 92, 129.

(3) Fuchs, N. Z. Phys. 1934, 89, 736 .

(4) Reerink, H.; Overbeek, J. Th. G. Discuss. Faraday Soc. 1954, 18
This is a transcendental equation for most potentials. Reerink and Overbeek ${ }^{4}$ have circumvented this rather uncomfortable situation by expanding $W(k)$ around a reference point $\kappa_{0}$ for one special case of the electrostatic potential. The reference point $\kappa_{0}$ was chosen such that the potential barrier $V\left(h_{\mathrm{g}}\right)$ vanishes. In the following we generalize their approach to arbitrary electrostatic potentials of the form $U(x, \kappa)$ where $x=\kappa h$. Using this form in eqs 1 and 4 we obtain

$$
A a_{K} / 12=-x_{s}^{2} U^{\prime}\left(x_{\mathrm{s}}, \kappa\right)
$$

where the prime denotes a partial differentiation with respect to $x$. This equation determines $x_{\mathrm{g}}=h_{\mathrm{g}} \kappa$ as a function of $\kappa$. At the reference point $\kappa_{0}$ introduced above $x_{8}$ has the value of $x_{0}$; the two quantities $\kappa_{0}$ and $x_{0}$ are determined by the following set of nonlinear equations

$$
\begin{gathered}
x_{0}=-U_{0} / U_{0}^{\prime} \\
a \kappa_{0}=12 x_{0} U_{0} / A
\end{gathered}
$$

The subscript indicates evaluation at $x_{0}$ and $\kappa_{0}$.

We now expand $\ln W$ into a Taylor series in $\ln \kappa$ around the reference point $\ln \kappa_{0}$, keeping terms up to first order

$$
\ln W \simeq \ln W_{0}+\left.\frac{\mathrm{d} \ln W}{\mathrm{~d} \ln \kappa}\right|_{0}\left(\ln \kappa-\ln \kappa_{0}\right)
$$

The zero-order term follows from inserting eqs 6 and 7 into eq 3 and can be expressed as

$$
W_{0}=\pi^{1 / 2} \frac{x_{0}}{2 a \kappa_{0}}\left(\beta U_{0}-x_{0}^{2} \beta U_{0}^{\prime \prime} / 2\right)^{-1 / 2}
$$

Since the linear coefficient in eq 8

$$
s_{0}=-\left.\frac{\mathrm{d} \ln W}{\mathrm{~d} \ln \kappa}\right|_{0}>0
$$

the stability ratio given by eq 8 decreases as $\kappa$ increases and becomes unity at

$$
\kappa_{\mathrm{c}}=\kappa_{0} W_{0}^{1 / s_{0}}
$$

The value of $\kappa=\kappa_{\mathrm{c}}$ determines the critical coagulation concentration. In several textbook discussions ${ }^{1}$ this condition is identified with $\kappa=\kappa_{0}$. As $\kappa_{0}>\kappa_{\mathrm{c}}$ this holds only in a first approximation.

Figure 1 explains the general features of the present approximation scheme for the calculation of stability ratios where $\ln W$ is plotted as a function of $\ln a \kappa$. The exact stability ratio shows a crossover from slow to fast aggregation with increasing $\alpha$ (thin line A). The thick line $B$ is the linear Taylor series approximation (eq 8) around the reference point $\kappa_{0}$. The value of the stability ratio at the reference point is given by eq 9 and the negative slope $s_{0}$. The critical $\kappa_{\mathrm{c}}$ given by eq 11 is located where the Taylor approximation equals zero.

Our analytical approximation of eq 2 can therefore be written as

$$
\ln W \simeq \begin{cases}-s_{0} \ln \left(\kappa / \kappa_{\mathrm{c}}\right) & \text { for } \kappa<\kappa_{\mathrm{c}} \\ 0 & \text { for } \kappa \geq \kappa_{\mathrm{c}}\end{cases}
$$

The two basic parameters $s_{0}$ and $\kappa_{\mathrm{c}}$ which enter eq 12 can be evaluated analytically. The slope $s_{0}$ is calculated by taking the derivative of eq 3 with respect to $x$ and making use of eqs 5-7. We obtain the result 


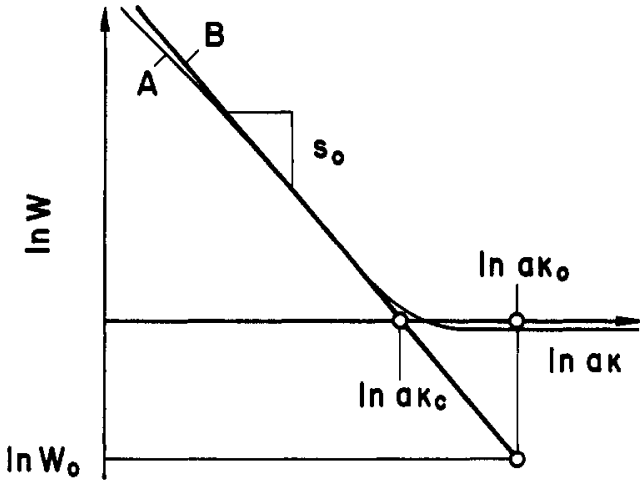

Figure 1. Schematic doubly logarithmic plot of the stability ratio $W$ for colloidal doublet formation as a function of dimensionless inverse Debye length $a_{\kappa}$ ( $a$ being the particle radius). Thin line $A$ is the exact stability ratio. Note that in the definition of $W$ we used $V(h)=0$ for the reference interaction. Since the van der Waals attraction is the actual interaction driving fast coagulation, in $W$ reaches a negative limiting value at high salt concentrations. Line $B$ is the present analytical approximation and is given by $\kappa_{c}$ which determines the critical coagulation concentration and its slope $s_{0}$.

$$
\begin{array}{r}
s_{0}=\beta U_{0}-\kappa_{0}(\partial U / \partial \kappa)_{0}+1+\frac{2 U_{0}-\kappa_{0} x_{0}{ }^{2}\left(\partial U^{\prime \prime} / \partial \kappa\right)_{0}}{2\left(2 U_{0}-x_{0}{ }^{2} U_{0}^{\prime \prime}\right)}- \\
\frac{\left[6 U_{0}+x_{0}{ }^{3} U_{0}^{\prime \prime \prime}\right]\left[U_{0}+x_{0} \kappa_{0}\left(\partial U^{\prime} / \partial \kappa\right)_{0}\right]}{2\left(2 U_{0}-x_{0}{ }^{2} U_{0}^{\prime \prime}\right)^{2}}
\end{array}
$$

By use of eqs 12 and 9 in eq 11 the critical coagulation condition can be shown to be

$$
\kappa_{\mathrm{c}}=\kappa_{0}\left(\frac{4 a^{2} \kappa_{0}^{2}\left(\beta U_{0}-x_{0}^{2} \beta U_{0}^{\prime \prime} / 2\right)}{\pi x_{0}^{2}}\right)^{-1 /\left(2 \varepsilon_{0}\right)}
$$

Equations 6, 7, 12,13, and 14 represent the central results of this note.

To illustrate the applicability of the general results, let use first consider the potential

$$
U(x, \kappa)=C e^{-x}
$$

where $C \propto a \tanh ^{2}\left(\beta \Phi_{\mathrm{g}} / 4\right)$. This interaction potential has been used in the study by Reerink and Overbeek. ${ }^{4}$ The solution of eqs 6 and 7 yields $x_{0}=1$ and $\kappa_{0}=12 \mathrm{C} /(e a A)$. Using these results and $\partial U / \partial \kappa=0$ in eqs 9,13 , and 14 , we obtain $W_{0}=\left[\pi e^{3} A^{2} /\left(288 \beta C^{3}\right)\right]^{1 / 2}$. The slope turns out to be

$$
s_{0}=\beta C / e-1 / 2 \simeq 0.37 \beta C-0.50
$$

whereas the critical coagulation concentration is determined by

$$
\kappa_{\mathrm{c}}=\frac{12 C}{e a A}\left(\frac{\pi e^{3} A^{2}}{288 \beta C^{3}}\right)^{1 /\left(2 s_{0}\right)} \simeq 4.42 \frac{C}{a A}\left(\frac{0.073 A^{2}}{\beta C^{3}}\right)^{1 /\left(28_{0}\right)}
$$

Note that by virtue of $a \kappa_{0} \gg 1$, the electrostatic coupling constant $C$ must be assumed to be much larger than the Hamaker constant $A$. Reerink and Overbeek ${ }^{4}$ have obtained for the slope $s_{0}=\beta C / e$, which is the leading term of eq 16. These analytical results agree well with numerical calculations (see Figure 2, graph A).

As a second example consider the Derjaguin approximation of the interaction potential obtained by solving the linearized Poisson-Boltzmann equation with constant potential boundary conditions ${ }^{1}$

$$
U(x, \kappa)=C \ln \left(1+e^{-x}\right)
$$

where $C \propto a \Phi_{\mathrm{g}}{ }^{2}$. An analogous calculation yields the

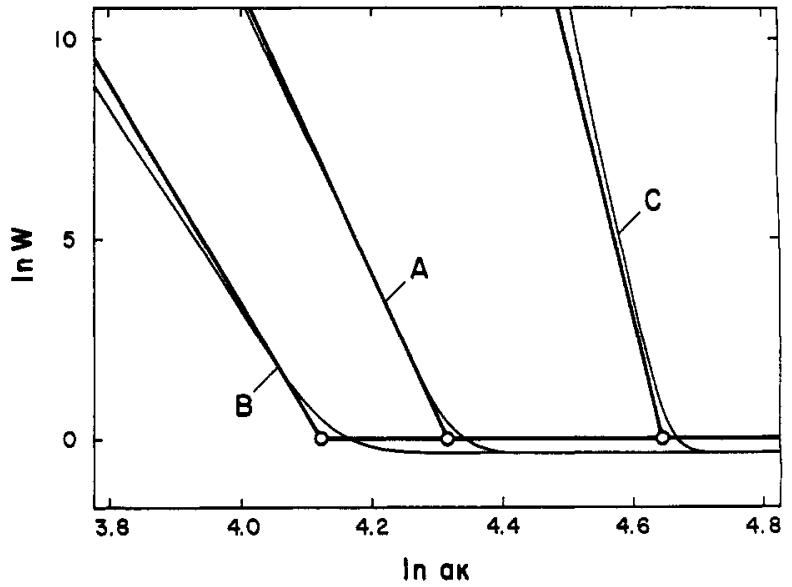

Figure 2. Comparison of exact stability ratios (thin lines) with the present analytical approximation (thick lines) for different interaction potentials: exponential potential eq $15(\mathrm{~A})$, constant electrostatic potential interaction eq $18(B)$, and constant charge interaction eq 21 (C). In all three cases $\beta A=5$ and $\beta C=100$. The barrier height at the critical concentration has the value $\beta V$. $\simeq 6$.

following slope and critical coagulation condition

$$
\begin{gathered}
s_{0} \simeq 0.28 \beta C-0.26 \\
\kappa_{\mathrm{c}} \simeq 3.79 \frac{C}{a A}\left(\frac{0.457 A^{2}}{\beta C^{3}}\right)^{1 /\left(2 s_{0}\right)} \quad(\text { for } C \gg A)
\end{gathered}
$$

A comparison with the exact result is shown in Figure 2 (graph B). The linear approximation intersects the exact stability curve at $\ln W \simeq 2$. The exact stability ratio has an inflection point at $\ln W \simeq 5$.

As the final example let us focus on the case of constant charge boundary condition ${ }^{1}$

$$
U(x, \kappa)=-C \ln \left(1-e^{-x}\right)
$$

where $C \propto a Z^{2}$. The results are

$$
\begin{gathered}
s_{0} \simeq 0.69 \beta C-1.51 \\
\kappa_{\mathrm{c}} \simeq 5.77 \frac{C}{a A}\left(\frac{0.053 A^{2}}{\beta C^{3}}\right)^{1 /\left(2 s_{0}\right)} \quad(\text { for } C \gg A)
\end{gathered}
$$

For this functional form of the interaction potential, the slope of our approximation (12) agrees well with the exact calculation (Figure 2, graph $\mathrm{C}$ ). The critical coagulation condition, however, is slightly underestimated and the linear approximation is shifted from the exact stability plot toward smaller values of $\ln \kappa$. It is interesting to note that this effect goes along with the lack of an inflection point in the exact stability plot.

An analogous treatment can be applied to the situation of reaction limited particle deposition in a flow field past spherical collectors. 6 Upon increasing ionic strength, a crossover from slow to fast deposition is observed which follows the same pattern as diffusive doublet aggregation. ${ }^{5}$ According to the analysis of Spielman and Friedlander the inverse collision efficiency $W^{(d)}$, which is defined as the ratio of fast and slow deposition rate constants, is given by 6,7

$$
W^{(d)} \simeq 0.6246\left(\frac{u}{D A_{\mathrm{g}} a_{\mathrm{c}}^{2}}\right)^{1 / 3} \int_{0}^{\infty}\left(f(h) \mathrm{e}^{\beta V(h)}-1\right) \mathrm{d} h
$$

where $D$ is the particle's diffusion coefficient, $u$ the velocity

(5) Elimelech, M.; O'Melia, C. R. Langmuir 1990, 6, 1153

(6) Spielman, L. A.; Friedlander, S. K. J. Colloid Interface Sci. 1974, 46,22 
of the particle toward the collector of radius $a_{\mathrm{c}}, A_{8}$ a porosity-dependent dimensionless parameter, $, 6,5$ and $f(h)$ accounts for the hydrodynamic screening between particle and collector. This latter function can be approximated 7 by $f(h)=1+a / h$. With the steepest descent approximation applied to eq 24 , the deposition stability ratio takes the form of eq 12 if we make the following substitutions

$$
\begin{gathered}
W \rightarrow W^{(d)} \\
s_{0} \rightarrow s_{0}{ }^{(d)}=s_{0}-1+\frac{U_{0}+\kappa_{0} x_{0}\left(\partial U^{\prime} / \partial \kappa\right)_{0}}{2 U_{0}-x_{0}{ }^{2} U_{0}^{\prime \prime}} \\
\kappa_{c} \rightarrow \kappa_{c}{ }^{(d)}=\kappa_{0}\left(a \kappa_{0} B W_{0} / x_{0}\right)^{1 / s_{0}^{(d)}}
\end{gathered}
$$

where $W_{0}$ is given by eq 9 and $B=1.2491\left[a^{3} u /\left(a_{c}^{2} A_{8} D\right)\right]^{1 / 3}$. The parameters $x_{0}$ and $\kappa_{0}$ are again determined by the solution of eqs 6 and 7.

We can illustrate these general results with the potential given by eq 15 . The slope is

$$
s_{0}{ }^{(d)}=s_{0}=\beta C / e-1 / 2
$$

The critical deposition concentration is determined by

$$
\kappa_{c}^{(d)}=\frac{12 C}{e a A}\left(\frac{\pi e B^{2}}{2 \beta C}\right)^{1 /\left(2 s_{0}\right)}
$$

These equations should be compared with eqs 16 and 17 for the case of diffusive doublet aggregation. Note that for the exponential form of the electrostatic interaction potential, eq 15 , the slope $s_{0}{ }^{(d)}$ has the same value as for

(7) Dahneke, B. J. Colloid Interface Sci. 1974, 48, 520. diffusive doublet aggregation. On the other hand, the value for the critical deposition condition $k_{c}{ }^{(d)}$ can be either smaller or larger than the critical coagulation condition $\kappa_{c}$, depending on the relative size of $a / a_{c}$ and the Peclet number $\mathrm{Pe}=u a_{\mathrm{c}} / D$.

In summary we have presented an analytical approximation for the stability ratios for diffusive doublet aggregation and deposition of colloidal particles for arbitrary electrostatic interaction potentials. For interparticle separations and Debye screening lengths small compared to the particle radius, we give explicit expressions for the critical coagulation conditions and slope in the stability plot. These are precisely the same quantities as determined in the experiment. Note that our prediction for the critical coagulation concentration is smaller than the textbook result obtained from the condition of vanishing barrier height. In our case the height of the potential barrier at the critical coagulation concentration is of the order of several thermal energy units. By use of interaction potentials which include size, charge, and structural polydispersity, the present approach might be useful in elucidating the long standing problem that experimental stability ratios are, in contrast to available theories, insensitive to particle size.

Acknowledgment. The present work has been performed in the group of Professor $\mathrm{H}$. Sticher to whom we are indebted for continuous support and interest. We thank P. Schurtenberger and J. C. Westall for careful reading of the manuscript. This work has been supported by the Swiss National Science Foundation. 